\title{
Modelling and optimisation of ionisation gauges for magnetic nuclear devices
}

\author{
A. Scarabosio, P. Sauter and G. Haas \\ Max-Planck-Institut für Plasmaphysik, EURATOM-Associaton, Boltzmannstr. 2, D-85748 Garching, Germany
}

\begin{abstract}
We have developed a numerical tool that simulates the operation of an ionisation-based pressure gauge in the presence of magnetic field. In this paper we describe the physical model, the numerical implementation and the validation of the code against laboratory experiments using the so called ASDEX pressure gauge. We then show how this modelling has led to a deeper understanding of processes associated with the presence of magnetic field in this type of gauge: sensitivity enhancement, sensitivity dependence on primary electron current and output saturation at high gas pressure. The combined electric and magnetic fields may trap electrons long enough so that they can spend all their energy in collisions with the neutral gas, thus increasing the sensitivity and leading to early saturation (with respect to the no B-field case). Although it seems difficult to avoid particle trapping with strong B-field, the fraction of trapped electrons and the trapping time can be limited by careful tailoring of the electric potential in the gauge and thus creating $E \times B$ drifts. This results in a modified sensitivity and saturation characteristic. Finally we show an example of a modified gauge which has an improved upper pressure limit in view of the next generation nuclear fusion experiment such as ITER.
\end{abstract}

Key words: ion gauge, neutral density, magnetic confinement, nuclear fusion

PACS: 07, 29, 34

\section{Introduction}

Neutral gas particle and/or flux density measurements in magnetic nuclear fusion experiments are important for the control and safe operation of devices such as tokamaks and stellarators. They are also fundamental for the understanding of the plasma-wall interaction and its influence on the plasma performance. Such measurements, however, are generally challenging. To achieve sufficient temporal and spatial resolution, several pressure gauges have to be installed inside the vacuum vessel. A suitable gauge has thus to work in presence of a strong, variable (in magnitude and direction) magnetic field and a noisy environment, and should provide reliable measurements for a typical pressure range of $10^{-7}-10^{-1}$ mbar. Under these conditions a standard hot cathode Bayard-Alpert ionisation gauge [1] cannot generally be employed. On the contrary, the so-called ASDEX Pressure Gauge (APG) [2] has been used to provide neutral flux measurements in previous and present-day tokamaks and stellarators such as ASDEX Upgrade, DIIID, JET and W7-AS.

The APG is also a hot cathode ionisation gauge. A schematic of the geometry is shown in figure 1 . The neutral particle density is derived from the electron (from the cathode) and ion (from ionisation) currents at the electrodes. The electron current is normally stabilised and kept constant by a feedback loop. In difference to standard ion gauges the APG uses a thick filament (as cathode), a linear geometry with the axis roughly parallel to magnetic field, and AC operation to cancel large offsets

Email address: andrea.scarabosio@ipp.mpg.de (A. Scarabosio) and leakage currents. More details can be found in reference [2]. The APG is also foreseen as the main in-vessel pressure gauge in ITER [3], but it must be optimised in order to meet ITER requirements, such as the pressure range [4]. We have thus started a theoretical investigation and implemented a numerical model that can be applied to all kinds of ionisation gauges with and without magnetic field.

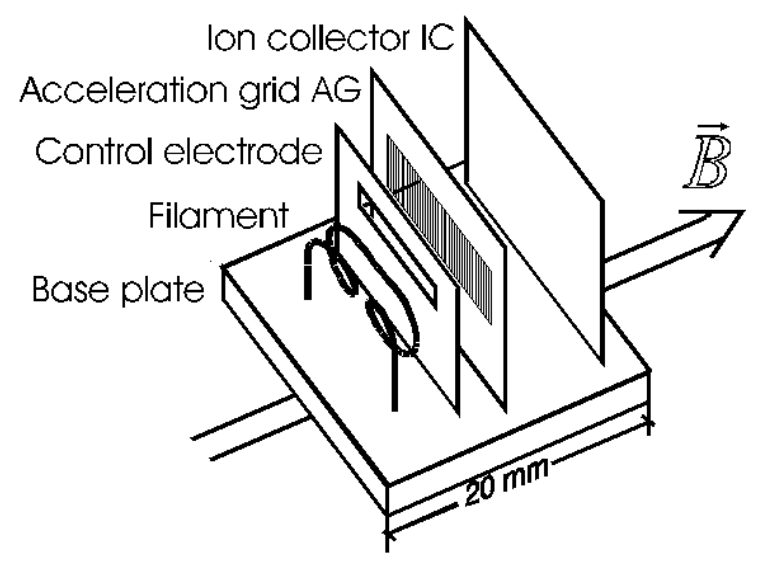

Figure 1: Schematic of the gauge head of an ASDEX pressure gauge (APG). The control grid allows AC operation by setting its voltage higher and lower than the filament potential.

\section{Numerical model}

We calculate the electric current at the electrodes, namely the electron current, $I_{e}$, to the acceleration grid and the ion current, 
$I_{i}$, to the ion collector by direct Monte Carlo methods. Specifically we follow the electrons leaving the source (such as a hot filament) by solving the single particle equation of motion subject to the Lorentz force, simulate their collisions with background neutral gas and count them when they are collected at the electrodes. We account for:

1. motion of primary and secondary (from ionisation) electrons and ions in realistic gauge geometry and electromagnetic field under steady state condition.

2. several elastic and inelastic interactions of electrons and ions with the neutral gas particles.

3. collective effect on trajectories from the (approximated) space charge of the electron beam.

We neglect:

1. other types of electron-electron interaction (such binary collision and beam instability), electron and ion interaction with electrode surfaces and radiation effects.

2. neutral particle motion $\left(\mathrm{v}_{e}>>\mathrm{v}_{\text {neutrals }}\right)$.

The electrons are initialised with a Maxwellian energy distribution (with a typical temperature of a few eV) and a user-defined spatial distribution (i.e. a function of the filament temperature in case of thermionic emission). Special care has been devoted to the choice of the trajectory integrator. Given the possibility of very long residence time (up to millions of gyro orbits and thousands of oscillations around the acceleration grid) in magnetic field, a very good energy conservation is crucial for a correct simulation. We found the best compromise between speed and energy conservation using a second order symplectic Verlet scheme [5]. The name symplectic integrator is given to a numerical scheme for the approximate solution of a hamiltonian system of differential equations. Its main advantage over other common integrators (i.e. Runge-Kutta) is the conservation of the hamiltonian and angular momentum [6]. At present, the gauge geometry can be arbitrarily modelled using planar and cylindrical objects (see fig. 2). It is foreseen to import directly $3 \mathrm{D}$ CAD drawings. The electrostatic and magnetostatic fields are computed by solving the 3D Poisson and Ampère equations with the finite element code ANSYS [7]. Appropriate boundary conditions are applied on the base plate, the electrodes and the computational boundaries (representing for instance the vacuum or a metallic box enclosing the gauge head). The field vectors are saved on a fixed grid and interpolated during the particle path integration. Alternatively, for simple geometries, a 'capacitor-like' (uniform between electrodes and null outside) analytic field model is used. We take into account both the main external guiding magnetic field and the filament field. The collisions are treated stochastically according to reference [8]. The cross-section data includes several processes in electron-atom/molecule impact such as elastic scattering, ionisation, excitation (orbital, vibrational and rotational), and attachment. The data has been collected from several databases [9]. For simplicity, the electron scattering is presently treated as isotropic but differential cross-sections could be straightforwardly included. Cross-section data for Argon, molecular Hydrogen, Neon and Helium has been implemented in the code.
The so-called 'null collision' technique is used to stochastically choose the time between collisions [10]. Secondary electrons from ionisation are initialised with a random energy chosen by the experimental distribution in reference [11]. Other distributions such as uniform $\left(E_{s e c}=R E_{\text {primary }}\right.$ with $R$ a pseudorandom number uniformly distributed in $[0-1]$ ) or 'equally shared energy' (50\%-50\%) have been tested with little variation of the results. To achieve a statistical uncertainty of about $5 \%$ we simulate between 10000 and 2 million primary electrons in order to get at least 1000 ionisations. The code has been implemented in Fortran 90 and a typical pressure scan (20 steps in the range of $10^{-4}-3 \cdot 10^{-1}$ mbar) takes from 80 to 500 minutes on a single CPU depending on configuration and background gas [12].

\section{Model validation}

The code is compared and validated against experimental data with several gases and with and without magnetic field. The model geometry implemented for this comparison is shown in figure 2 whereas the potentials applied to the electrodes and the potential variation along the gauge axis are sketched in figure 3. We note that the physical model applied here has no free parameters in the sense that all geometric and electromagnetic values are fixed and taken from actual measurements.

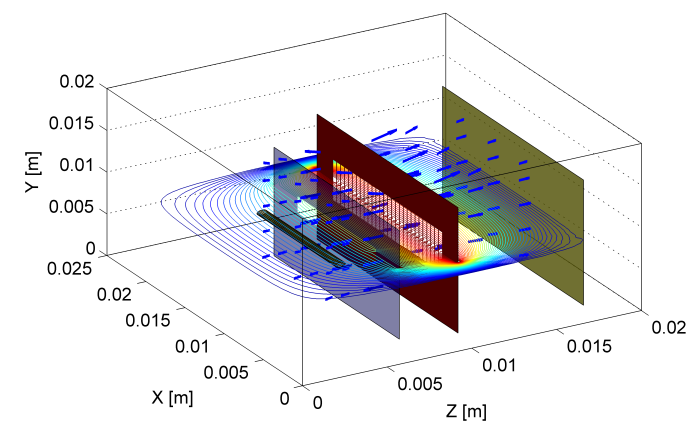

Figure 2: Computational box and model geometry of the APG's electrodes and electrostatic field. The filament is approximated by a straight cylinder in front of the control grid slit and its contribution of the heating current to the magnetic field is also taken into account. The arrows show the electrostatic field from finite element calculations. Continuous lines represent a bi-dimensional contour plot of the potential.

\subsection{Simulation versus experiment: no B-field case}

We plot the gauge characteristic in terms of the normalised output, $I_{i} /\left(I_{e}-i_{i}\right)$, versus pressure. Without magnetic field the APG behaves linearly as any standard ionisation pressure gauge. The output from the simulation compares quite well with experiments with a quantitative agreement within $30-40 \%$ (figure 4) for a pressure range spanning about 4 orders of magnitude. We extract the constant sensitivity, $I_{i} /\left(I_{e}-i_{i}\right) / p$, by applying a linear regression on both experiments and simulations and compare it with established data in table 1. 


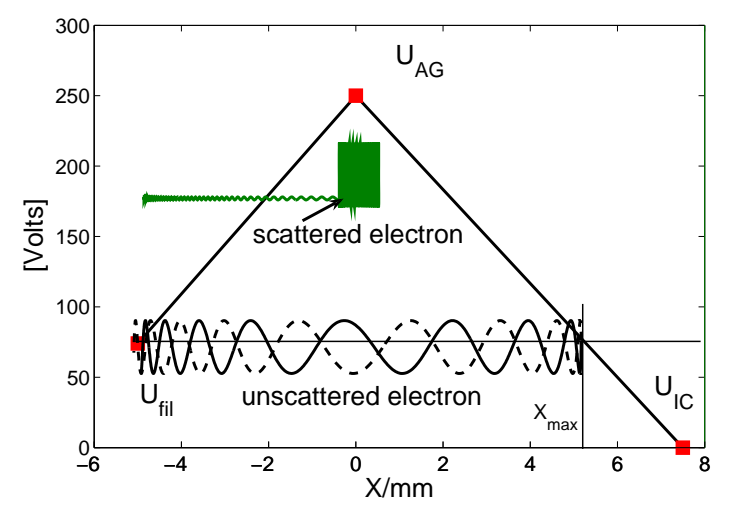

Figure 3: Sketch of the electrostatic potential of the APG. The acceleration grid at $\mathrm{z}=0$ is at $250 \mathrm{~V}$, control grid $105 \mathrm{~V}$, filament $70 \mathrm{~V}$ and the ion collector is grounded together with the base plate. One can clearly seen that the electrons move in a potential well in which may be trapped. We illustrate paths of a trapped (scattered) and untrapped (unscattered) electron. Larmor radii are exaggerated for clarity.

Table 1: Relative sensitivity comparison of experiment, simulation and literature [13]

\begin{tabular}{|c|c|c|c|}
\hline & $\mathrm{Ar} / \mathrm{H}_{2}$ & $\mathrm{Ne} / \mathrm{H}_{2}$ & $\mathrm{He} / \mathrm{H}_{2}$ \\
\hline Literature & 2.98 & 0.55 & 0.35 \\
\hline Experiment & 2.68 & 0.55 & 0.30 \\
\hline Simulation & 2.83 & 0.58 & 0.35 \\
\hline
\end{tabular}

The relative sensitivities for the different gases are in good agreement showing the high quality of the cross-section data. Without strong guiding field the gauge characteristic is quite insensitive to the details of the electromagnetic structure (boundary and edge effects) because of the short residence time of the electrons (time spent in the ionisation volume).

\subsection{Simulation versus experiment: B-field at 2 Tesla}

When introducing the guiding magnetic field the dynamic changes dramatically. The electrons, confined by the magnetic field and moving in the potential well (figure 3), may either return to the filament due to their initial energy [2], or get trapped if its parallel momentum changes during the first transit (i.e. scattered by a collision). These trapped electron dominate the ionisation process. A typical path from the filament to the ionisation volume and back takes about 10ns while a trapped electron can make more than 1000 transits. For the same pressure, due to this long trapping time, the output increases by more than one order of magnitude when increasing B from 0T to about 1T. This effect can be clearly seen in the experimental data (full line with circle symbols in figure 3.2) carried out with an electron current of $20 \mu \mathrm{A}$ and $2 \mathrm{~T}$ magnetic field (see also references [2] and [4]).

The enhanced gauge output is also reproduced by the numerical model indicated as 'simple' in figure 5 (dash-dotted line with triangles). This model considers the electrostatic field produced by large plates with no grids or slits. By statistic analysis

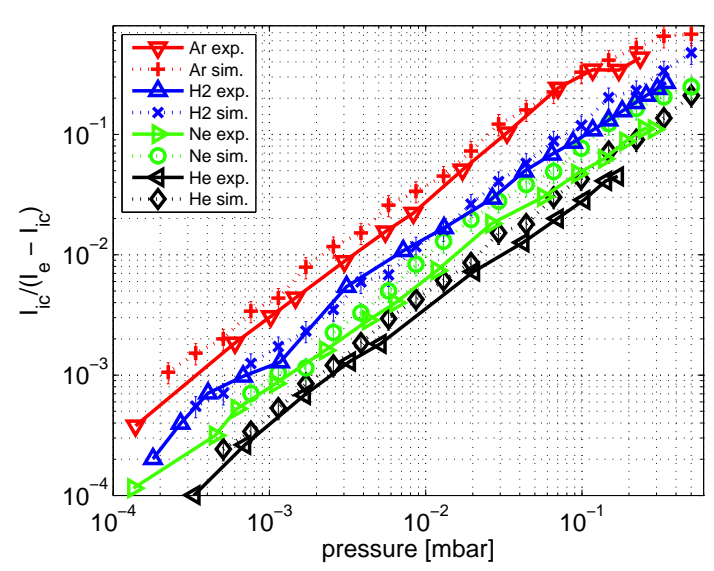

Figure 4: Comparison of simulation (dotted) with experiment (solid) for Argon, Hydrogen, Neon and Helium with no guiding field and uniform thermionic from the filament.

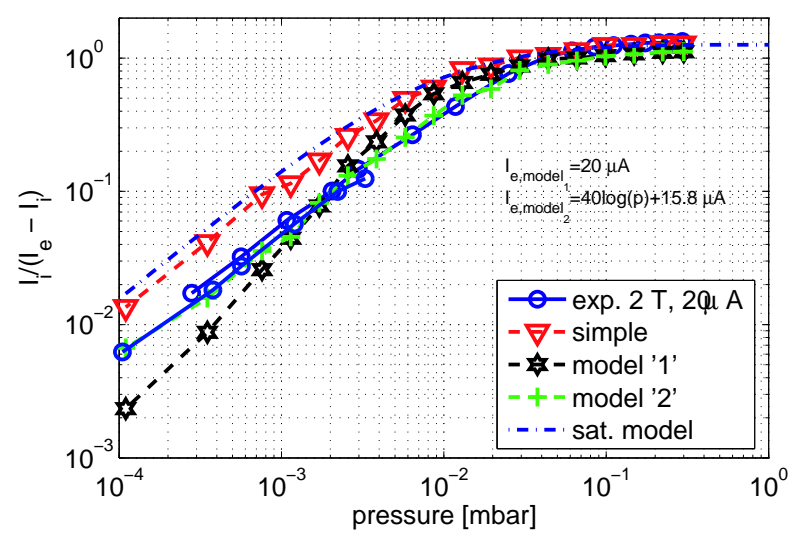

Figure 5: Comparison of simulation (dotted lines) with experiment (solid lines) for $\mathrm{H} 2$ gas and $2 \mathrm{~T}$ guiding B-field. Triangles are calculated with analytic capacitor-like E-field. Stars and crosses include the effect of the electron space charge respectively constant and variable with pressure and finite-size electrodes effects. The dash-dotted line with no symbols is calculated with ea. 3.

of the trajectories and collisions during the simulation we find two basic reasons for this enhancement:

$i$ the increased fraction of electron current returning to the filament, $I_{b k f i l}$, reduces the current measured at the acceleration grid, $I_{e}$, which in turn increases the normalised output.

ii The electrons ionise up to 6 times before getting absorbed at the electrodes due to their long trapping time (whereas without guiding field they ionise typically once or twice maximum).

The other important aspect is the early saturation occurring at pressure of few $10^{-2}$ mbar, earlier than without B-field. This feature is also well described by the simulation and will be treated in more detail in the next section.

At low pressures the result of this 'simple' electromagnetic model may be 2-3 times larger than the experiment. This differ- 
ence can be reduced by considering a more detailed electromagnetic model (EM) including boundary and space charge effects (see model '1' and '2' in fig. 5). In particular model '1' includes finite size electrodes, the base plate, and a uniform beam-spacecharge from the filament emission. The space charge density is estimated as $n_{e}=4 I_{e} /\left(e \mathrm{v}_{\max } S\right)$, where $I_{e}$ is taken from the experiment, $S$ is the projected surface of the filament, $\mathrm{v}_{\max }$ is the maximum unperturbed velocity at the acceleration grid and $e$ the electron charge. Model '2' achieves a very good agreement by using a pressure dependent (increasing) charge density. This is qualitatively justified by considering the presence of an increasing fraction of secondary and trapped electrons [4] with pressure but the function $n_{e}(p)$ used here is however arbitrary. It is important to realise that all these additional effects create an electric field component non parallel to B (i.e. vertical) and thus $E \times B$ drifts. To see how these drifts affect the dynamic let's consider an $e^{-}$born on a field line passing through the grid which gets trapped because of a collision (elastic or inelastic) during the first transit. For the magnetic field of interest (small Larmor radius) the scattering with neutrals is very ineffective in detrapping the electron which can then ionise until its energy goes below the threshold (limit of infinite $t_{\text {trapping }}$ ). In the presence of the $E \times B$ drift, however, the electrons slowly reach a magnetic field line intersecting the grid before having consumed all the available energy (limited $t_{\text {trapping }}$ ). In the 'simple' EM model there is no $E \times B$ drift (E purely parallel to $\mathrm{B}$ ), whereas in the other models it exists. This explains the higher output at low pressure in the 'simple" model. All models show a very similar saturation which is in good qualitative agreement with the experiment. This is because, at high pressure, the characteristic energy loss time becomes short compared with the trapping time, regardless of drifts. Despite the difficulty of accurately describing the potential in the gauge in order to quantitatively match the experiment, it is clear that the main ion gauge physics is included in the simulations (even for the simple EM model). To develop further physical intuition we next attempt an analytical description of the saturation mechanisms.

\subsection{Saturation model}

We start by writing the currents $I_{e}$ and $I_{i}$ in the following way:

$$
\begin{aligned}
I_{i} & =f \cdot I_{s e c}(p) \\
I_{e} & =I_{\text {emit }}+I_{\text {sec }}(p)-I_{b k f i l}(p) \\
I_{\text {sec }} & =\sum_{i} I_{\text {sec }}^{i}
\end{aligned}
$$

where $I_{s e c}(p)$ is the current from secondary electrons (first, second, etc ... generation) which is a function of pressure $p . f$ is the fraction of secondary electrons (and thus ions) produced in the ionisation volume (i.e. between acceleration grid and ion collector) which depends mainly on the gauge geometry. For the standard gauge $f \sim 0.65$. $I_{\text {emit }}$ is the current emitted from the filament and passing through the control grid. It is constant for constant heating current and emission condition. $I_{b k f i l}$ is the current of electrons returning to the filament. Up to this point the formulation is quite general. Now we assume the following physical picture: an electron born on a magnetic field line passing through the grid becomes trapped if it is scattered (even just a single elastic collision). If it does not collide with any neutrals, it will then return to the filament and be absorbed. The trapped electron current, $I_{\text {trap }}$, and $I_{b k f i l}$ can then be written as:

$$
\begin{aligned}
I_{\text {trap }}(p) & =I_{\text {emit }}-I_{1 \text { pass }}-I_{\text {bkfil }}=I_{\text {emit }} \cdot t\left[1-\exp \left(-\alpha \frac{p}{k T}\right)\right] \\
I_{1 \text { pass }} & =(1-t) I_{\text {emit }} \\
I_{b k f i l}(p) & =I_{\text {emit }} \cdot t \exp \left(-\alpha \frac{p}{k T}\right) \\
\alpha & =\oint \sigma_{\text {tot }}(v) d x
\end{aligned}
$$

where $k$ is the Boltzmann constant and $\sigma_{\text {tot }}$ is the total scattering cross-section. The integral $\alpha$ is performed along the electron path from the filament to the ionisation volume and back. $p / k T$ gives the neutral particle density at gas temperature $T$. The exponential factor represents the probability of having no collision on the integration path. The transparency of the acceleration grid is indicated by $t$ and, in the example considered in figure 3 , is equal to about $80 \%$. $I_{1 \text { pass }}$ are the electrons starting on field lines intersecting the acceleration grid which are absorbed at their first pass. For standard gauge geometry and potential one finds $\alpha=8.4 \cdot 10^{22} \mathrm{~m}^{3}$. The trapped electrons ionise the neutral gas once or several times depending on their tapping time. Let us define $n_{\text {ion }}^{1}$ as the average number of ionisations per primary trapped electron. Then we have $I_{\text {sec }}^{1}=n_{\text {ion }}^{1} I_{\text {trap }}$. In general $n_{\text {ion }}^{1}$ will depend on pressure. We can make the simplifying assumption that the trapping time is long enough to allow the electron to spend all its energy in inelastic collisions. With this assumption $n_{i o n}^{1}$ is constant and depends only on the accelerating field and gas type. Similarly we have for the tertiary, quartiery, etc ... electrons $I_{\text {sec }}^{i}=n_{\text {ion }}^{i} I_{\text {sec }}^{i-1}$.

Combining the above definitions and equations (1-2) the normalised output can be written:

$$
\begin{aligned}
\frac{I_{i c}}{I_{e}-I_{i c}} & =f \cdot \kappa \cdot t \frac{1-\exp \left(-\alpha \frac{p}{k T}\right)}{1+(1-f) \kappa t-[(1-f) \kappa+1] t \exp \left(-\alpha \frac{p}{k T}\right)} \\
\kappa_{\text {ion }} & =\Sigma_{i} \Pi_{j}^{i} n_{i o n}^{j}
\end{aligned}
$$

With only $\kappa_{i o n}$ left as a free parameter we fit the 'simple' simulation in fig. 5 with eq. (3). $\kappa_{\text {ion }}=5.7$ yields a good agreement especially at high pressure. One can see that the linear regime of the ionisation gauge in magnetic field corresponds to the phase where only a small fraction of the electrons is actually trapped. The turning point is around $2 \cdot 10^{-2}$ mbar where $I_{\text {trap }} / I_{\text {emit }}$ surpasses $20 \%$ for this configuration. 


\section{Optimisation}

With the goal of extending the pressure range of the APG two main guidelines arise: i) decrease the fraction of trapped electrons for a given pressure by reducing electron scattering and ii) reduce the trapping time by increasing the $E \times B$ drift. Among the many possible practical solutions we show two examples of modified configurations. In both we accelerate the electrons earlier and reduce the ionisation volume $\left(U_{c g}\right.$ from 105 to $200 \mathrm{~V}$, ionisation volume from 7.5 to $5 \mathrm{~mm}$ ) with the effect of reducing the scattering (especially elastic scattering). In the first example we additionally reduce the transparency of the acceleration grid from 80 to $50 \%$, whilst in the second we add an horizontal electrode plate attached to the upper part of the ion collector which is also grounded to bend the electric field and increase the drifts. The results are shown in figure 6. In both cases the slope of the characteristic is still sufficient up to pressures above $10^{-1}$ mbar at the expense of a lower sensitivity (lower $I_{i}$ current). Further improvements may be expected with others modifications.

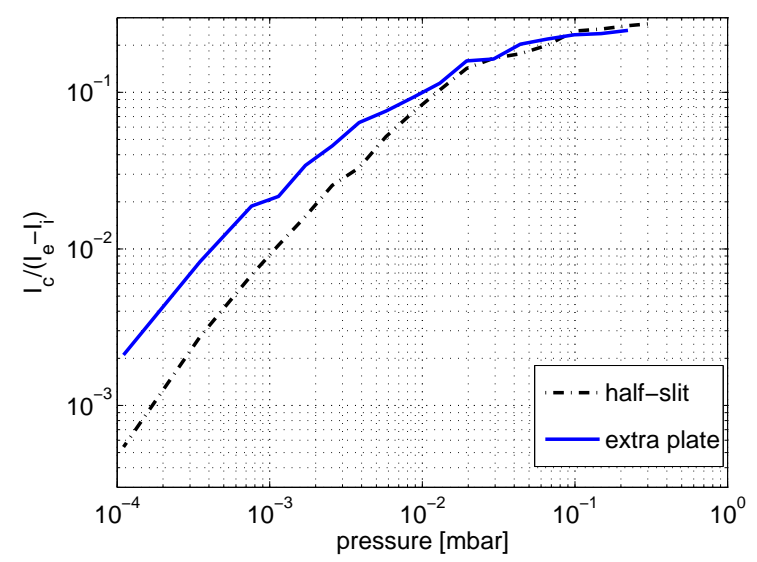

Figure 6: Modified APGs for higher pressure limit with $U_{c g}=200 \mathrm{~V}$ and $5 \mathrm{~mm}$ ionisation volume. The dash-dotted line has $50 \%$ transparency of the acceleration grid while the full line has extra horizontal plate attached to the upper part of the ion collector to 'curve' the electric field and increase the $E \times B$ drift.

\section{Conclusion}

We have developed a numerical model to describe ionisation pressure gauges in magnetic field. We have then validated it against dedicated laboratory experiments with and without magnetic field. This simulation tool can be used to optimise the parameters of an existing ion gauge and to develop new concepts. The analysis of the model results and the comparison with the experiments has led to a deeper physical picture of the ASDEX gauge in presence of magnetic field. Although the 'single particle' picture so far adopted seems adequate, for an accurate quantitative prediction extreme care must be devoted to the modelling of the electromagnetic field inside the gauge including boundary and space charge effects.

\section{References}

[1] R. T. Bayard and D. Alpert, J., REv. Sci. Instr. 21, 572 (1950)

[2] Haas G., Bosch H.-S., Vacuum 51 (1998), p. 39-46.

[3] Mertens V., Haas G., In Vessel Neutral Pressure Measurement in ITER, Final Report on EFDA Contract 5-1349 Deliverable D 4.1, 2006

[4] A. Scarabosio and G. Haas, AIP Varenna Conference Proceedings, 998(238), 2008

[5] L. Verlet, Phys. Rev., 159(1):98, 1967.

[6] E. Hairer, C. Lubich, and G. Wanner. Geometric Numerical Integration: Structure-Preserving Algorithms for Ordinary Differential Equations. Springer Series in Computational Mathematics. Springer, 2006.

[7] ANSYS, Commercial Finite Element Computation Package, ANSYS Inc, Canonsburg US.

[8] G.W. Fraser, E. Matheison, Nucl. Instr. and Meth. A 247 (1986) 544

[9] S.F. Biagi, Nucl. Instr. and Meth. A 421 (1999) 234-240

[10] H.R. Skullerud, Brit. J. Appl. Phys. (J. Phys. D) 1 (1968) 1567

[11] S. Yoshida and A. V. Phelps, Phys. Rev. A, 27(6):2858, 1983

[12] P. Sauter, Diploma Thesis, LMU Muenchen 2008, http://edoc.mpg.de

[13] Leybold Vakuum GmbH., Ionivac manual 